Research Article

\title{
Evaluation and Application of Surrounding Rock Stability Based on the Improved Weighting Multidimensional Cloud Model
}

\author{
Jing Long Li $\mathbb{D},{ }^{1,2}$ Chao Yuan $\left(\mathbb{D},{ }^{1}\right.$ Bo Zhang, ${ }^{1}$ and Bin Sui ${ }^{1}$ \\ ${ }^{1}$ School of Civil Engineering Civil Engineering Building, Qianfoshan Campus Shandong University, 17923 Jingshi Rd, \\ Jinan 250061, China \\ ${ }^{2}$ Key Laboratory of Geological Hazards on Three Gorges Reservoir Area (China Three Gorges University), Ministry of Education, \\ China Three Gorges University, Yichang, Hubei, China \\ Correspondence should be addressed to Chao Yuan; chao.yuan@sdu.edu.cn
}

Received 22 February 2021; Revised 6 May 2021; Accepted 29 May 2021; Published 6 July 2021

Academic Editor: Xiaoli Liu

Copyright () 2021 Jing Long Li et al. This is an open access article distributed under the Creative Commons Attribution License, which permits unrestricted use, distribution, and reproduction in any medium, provided the original work is properly cited.

\begin{abstract}
Aiming at the uncertainty in the existing tunnel surrounding rock stability evaluation methods and the inability to make scientific explanations in the process of determining the weights of multiple evaluation indicators, this paper combines subjective weighting based on the nonlinear characteristics of the surrounding rock stability evaluation criteria. Based on the advantages of the method AHP and the objective weighting method CRITIC, a comprehensive weighting method with multiple evaluation indicators is proposed, which is integrated with the multidimensional normal cloud model to establish a surrounding rock stability evaluation method for the improved weighted multidimensional cloud model and accurately obtain the safety level of tunnel surrounding rock construction. The multidimensional cloud model based on improved weighting not only solves the two major problems of randomness and ambiguity in surrounding rock stability evaluation but also fully considers the influence of the information contained in each evaluation factor on the results. The surrounding rock stability evaluation method in this paper is applied to the tunnel construction of the Beijing-Shanghai Expressway and Jinan Expressway connecting line construction project, and the evaluation results have a scientific guiding role for construction risk control.
\end{abstract}

\section{Introduction}

Because of the complex bad geology in the surrounding rock of the tunnel and existence of many uncertain factors, it is easy to collapse during the construction of the tunnel, and the risk is very high. Generally speaking, the better the evaluation and classification of surrounding rock stability, the lower the probability of safety accidents during construction. The evaluation of risk grade of tunnel construction safety should be based on reasonable surrounding rock stability grade evaluation, before tunnel design and construction. It is necessary to make scientific evaluation on surrounding rock stability grade according to the relevant rock mass quality index [1-3].

With the rapid development of urbanization and the continuous growth of the population, underground engineering has been paid more and more attention. Its scale and quantity have also increased rapidly, and the engineering environment faced by the project is becoming more and more complex. Classification of surrounding rock stability is directly related to the economic rationality, safety, reliability, and even success or failure of the project, so the classification of surrounding rock stability is an important problem, but the evaluation of surrounding rock stability is extremely complex, involving many uncertain influencing factors [4]. For this reason, people have established various evaluation methods based on different theories, such as the variable fuzzy set method, rough set method, artificial neural network method, extension method, stochastic simulation method, and cloud theory method, which are applied in engineering practice and achieved useful progress. For example, Xue and Xiao proposed a least square support vector machine (LSSVM) method based on particle swarm optimization [5], Wu et al. proposed the stability evaluation of 
the underground engineering surrounding rock based on DS evidence theory and error elimination theory model [6], and Ren et al. established the rock mass deformation stability prediction evaluation method (DSPEM), which combines the safety evaluation method based on deformation statistical analysis with the antideformation prediction method based on measured deformation data [7]. Ran et al. used the multidimensional normal cloud model as a rockburst grade prediction method, used rough sets to determine the evaluation index weights, and established a rough set-multidimensional normal cloud rockburst classification prediction model [8]; Zhong et al. and Wang et al., aiming at the multiple indicators and incompatibility of tunnel surrounding rock stability evaluation, combined the game theory with subjective and objective weighting methods, combined with the cloud model theory to improve the extension theory, and established a game for the stability evaluation of the tunnel surrounding rock on the extension cloud model $[9,10]$. However, these methods also have shortcomings. For example, the actual application of artificial neural network methods is affected by the bottleneck of knowledge acquisition; the extension theory method will miss important constraints; the cloud model based on the normal distribution assumes the evaluation index distribution form is a normal distribution.

Evaluation of the stability classification of the surrounding rock is the coexistence of randomness and fuzziness, which is mainly manifested in three aspects:

(1) Multiple evaluation factors should be considered comprehensively in the evaluation process, and the classification of individual evaluation factors is a random event [11].

(2) The existence of evaluation factors in the boundary range is also ambiguous.

(3) The weight of each evaluation factor is not sure. In order to solve the above problems, this paper combines the subjective weighting method with the objective weighting method to determine the weight of each index in the evaluation of surrounding rock stability and establishes a comprehensive evaluation model of surrounding rock stability of the multidimensional normal cloud model.

\section{Multidimensional Normal Cloud Model Analysis Method}

2.1. Model of the Multidimensional Normal Cloud. In practical engineering, the surrounding rock evaluation language collected by current data information is often imprecise, fuzzy, and random, and even some indexes are only descriptive. Therefore, even if many index parameters of the surrounding rock of the face are known, it is often difficult to give an accurate classification of the stable grade of the surrounding rock. Based on the probability theory and fuzzy set, the cloud model evaluation method can be utilized to form the conversion model between the qualitative concept and quantitative representation. It serves to reveal the internal correlation between randomness and fuzziness of surrounding rock evaluation index information [12].

The cloud model is an uncertainty conversion model between a qualitative concept expressed in the natural language and its quantitative representation, in which the universality of the normal distribution and normal membership function lays a theoretical foundation for the universality of the positive cloud. Therefore, normal cloud has become the most widely used cloud model. The digital features of cloud models play an important role in the process of cloud model generation, which is mainly composed of expectation $E(x)$, entropy $E n$, and superentropy He, $E(x)$ representing the expectation that cloud droplets are distributed in the domain space in the cloud model; $E n$ reflecting the uncertainty measure of the domain space in the qualitative concept, indicating the possible distribution range of cloud droplets; and He representing the uncertainty and randomness of cloud droplets in the domain space distribution.

The multidimensional normal cloud model extends the one-dimensional cloud model to multidimensional [13], that is, the three digital features of the cloud model are multidimensional, and $W$ can represent multiple qualitative concepts or the multidimensional attributes of a qualitative concept. And to reflect the multidimensional qualitative concept, let $U$ be an $m$-dimensional domain $U=\left\{x_{1}, x_{2}, \ldots, x_{m}\right\}, T$ be a qualitative concept about $U$, and the membership degree of the elements in $U$ be a random number with a stable tendency $\mu$, that is,

$$
\begin{aligned}
& \mu: U \longrightarrow[0,1], \\
& \forall\left(x_{1}, x_{2}, \ldots, x_{m}\right) \in U,\left(x_{1}, x_{2}, \ldots, x_{m}\right) \longrightarrow \mu .
\end{aligned}
$$

Assuming that the dimensions of the universe are not related to each other, the $m$-dimensional normal cloud is described by $3 m$ digital features, including expectation $E x\left(E x_{1}, E x_{2}, \ldots, E x_{m}\right)$, entropy $E n\left(E n_{1}, E n_{2}, \ldots, E n_{m}\right)$, and superentropy $E n\left(E n_{1}, E n_{2}, \ldots, E n_{m}\right)$. The mathematical expected hypersurface (MEHS) equation of the multidimensional normal cloud model is

$$
\operatorname{MEHS}\left(x_{1}, x_{2}, \ldots, x_{m}\right)=\exp \left[-\frac{1}{2} \sum_{i=1}^{m} \frac{\left(x-E x_{i}\right)^{2}}{E n_{i}^{2}}\right] \text {. }
$$

The generation algorithm of the multidimensional cloud model based on this equation is called the cloud generator, which is divided into forward cloud generator and reverse cloud generator. The forward cloud generator is also called the basic cloud generator, and it is briefly described. The generator algorithm is as follows:

(1) Generate $k x_{i}=\left(x_{1}, x_{2}, \ldots, x_{m}\right)$-dimensional normal random numbers with $E x\left(E x_{1}, E x_{2}, \ldots, E x_{m}\right)$ as the expectation and $E n\left(E n_{1}, E n_{2}, \ldots, E n_{m}\right)$ as the variance.

(2) Generate $k y_{i}=\left(y_{1}, y_{2}, \ldots, y_{m}\right)$-dimensional normal random numbers with $E n\left(E n_{1}, E n_{2}, \ldots, E n_{m}\right)$ as the expectation and $\mathrm{He}\left(\mathrm{He}_{1}, \mathrm{He}_{2}, \ldots, \mathrm{He}_{m}\right)$ as the variance. 
(3) Calculate the membership degree:

$$
\mu_{i}=\exp \left[-\frac{1}{2} \sum_{i=1}^{m} \frac{\left(x-E x_{i}\right)^{2}}{E n_{i}^{2}}\right] \text {. }
$$

(4) Drawing a multidimensional cloud drop model, $\left(x_{i}, y_{i}, \mu_{i}\right)$ is the cloud drop.

Taking the cloud model generated by the forward cloud generator as an example, the calculation results of the cloud model with the above process are repeated. Figure 1 shows the calculation results of the single-factor one-dimensional cloud model and the two-factor two-dimensional cloud model.

The two-dimensional forward cloud model is a highdimensional cloud model that can be presented in a threedimensional graph by mapping software. When more multidimensional indicators are considered at the same time, the cloud model can only be expressed by formula, and the corresponding degree of certainty can be calculated by program. In order to further explain the membership determination process of $2 \mathrm{D}$ and high-dimensional cloud models in hierarchical applications, the four evaluation ranges of a two-factor index are used to draw the cloud models with the four standard grades for the evaluation of $2 \mathrm{D}$ cloud models.

It can be seen from Figure 2(b) that the multidimensional cloud model has the advantages of fast and intuitive in determining the level of membership of multiple indicators. At the same time, the high-dimensional cloud model generated by considering the correlation between multiple factors can more reveal the internal correlation of the evaluation target.

\subsection{AHP-CRITIC Empowerment Method}

2.2.1. AHP Method to Determine Weight. The analytic hierarchy process (AHP) is a decision-making method that combines qualitative and quantitative analyses. In the process of determining weights, expert opinions are used as the basis for evaluation, and the results can fully apply the experience of industry experts to evaluate the stability of surrounding rock. It is a subjective weighting method with the advantages of systemicity and flexibility. This method divides various indicators in the complex surrounding rock stability evaluation problem into orderly levels related to them for quantitative and qualitative analyses. Table 1 shows the rules for the relative importance of evaluation indicators in the analytic hierarchy process.

After determining the relative importance judgment matrix, formulas (1)-(3) are used to calculate

$$
\begin{aligned}
& m_{i}=\prod_{j=1}^{n} x_{i j}, \quad(i, j=1,2, \ldots, n), \\
& \bar{w}_{i}=\sqrt[n]{m_{i}} .
\end{aligned}
$$

Normalization calculation is performed on the evaluation index weight vector to determine the weight of a single index:

$$
w_{i}=\frac{\bar{w}_{i}}{\sum_{j=1}^{n} \bar{w}_{j}} .
$$

Finally, a consistency test is carried out to check whether the judgment matrix is consistent, which needs to satisfy $a_{i k}=a_{i j} \times a_{i k}$ for any evaluation of surrounding rock stability index $i, j$, and $k$.

2.2.2. CRITIC Determines the Weight. CRITIC (criteria importance through intercriteria correlation) is a method of showing the importance of indicators by analyzing the correlation between indicators. As an objective weighting method, contrast strength and indicator conflict are the basis for determining objective weights [14]. In the evaluation of the surrounding rock stability index of this article, the contrast intensity refers to the standard deviation of each surrounding rock evaluation index applied to different surrounding rock conditions. The larger the standard deviation, the greater the amount of information contained in the index. The conflict of indicators is based on the correlation of indicators. If the negative correlation coefficient of an indicator is larger, it means that the information reflected between the indicators is different, and its weight will be greater.

The CRITIC method not only considers the amount of information of the indicators but also considers the correlation between the indicators, which has obvious objective advantages. By unifying the dimensions and orders of magnitude among the indicators, the optimization and improvement of the use of standard deviation to measure the variability of indicators are insufficient. The main steps are as follows: (7)-(10).

Coefficient of variation of evaluation indicators:

$$
v_{j}=\frac{s_{j}}{\bar{x}_{j}}, \quad(j=1,2, \ldots, n),
$$

where $v_{j}$ is the coefficient of variation of the $j$ index and $s_{j}$ and $\bar{x}_{j}$ are the mean value and standard deviation of the $j$ index, respectively.

Calculate the correlation coefficient matrix:

$$
R=\left(r_{k l}\right)_{n \times n}, \quad(k=1,2, \ldots, n ; l=1,2, \ldots, n),
$$

where $r_{k l}$ is the correlation coefficient between the $k$ index and the $l$ index.

Taking $C_{j}$ as the amount of information contained in the $j$ indicator, the formula for determining $C_{j}$ is as follows:

$$
C_{j}=s_{j} \sum_{j=1}^{m}\left(1-r_{k j}\right), \quad(j=1,2, \ldots, m) .
$$

In the formula, $\sigma_{j}$ is the standard deviation of the $j$ indicator, and $r_{k l}$ is the correlation coefficient of the $i$ and $j$ indicators. According to the information reflected in the 


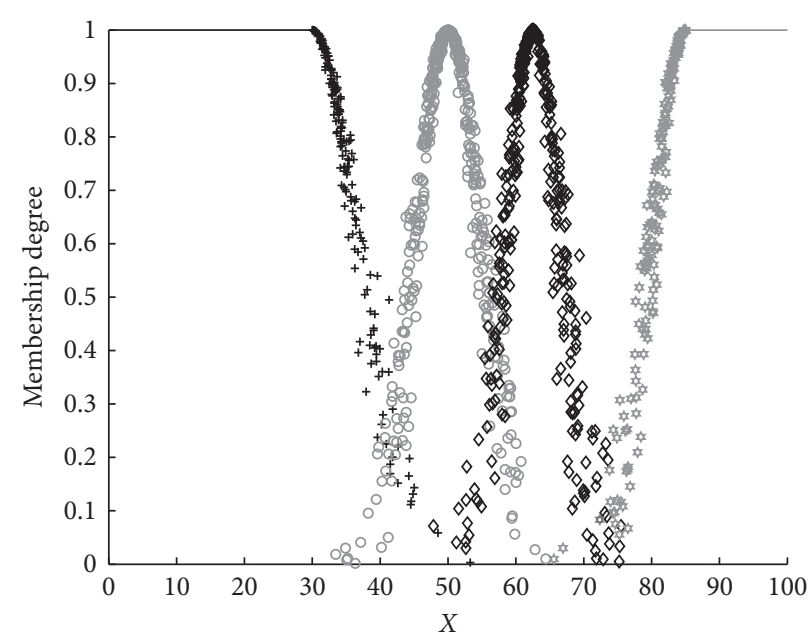

(a)

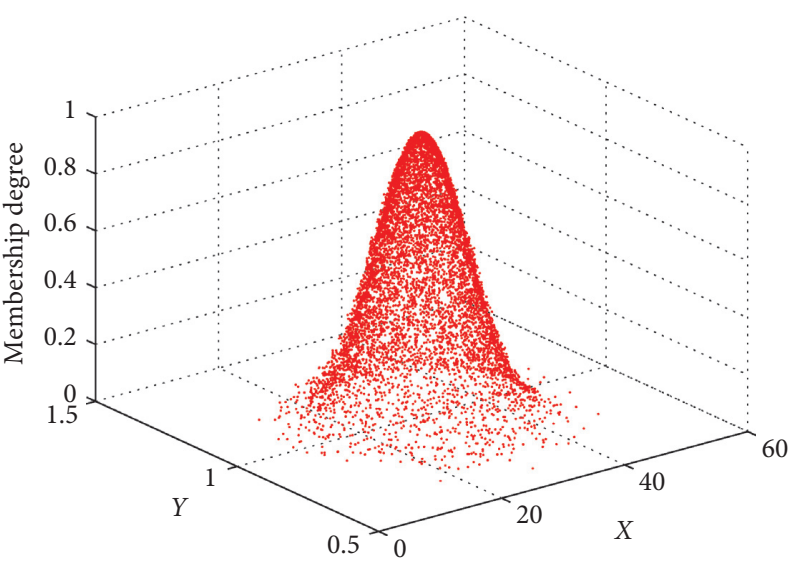

(b)

Figure 1: $X-Y$ two-factor qualitative conceptual 2D cloud model. (a) Single-factor one-dimensional cloud model. (b) Two-factor twodimensional cloud model.

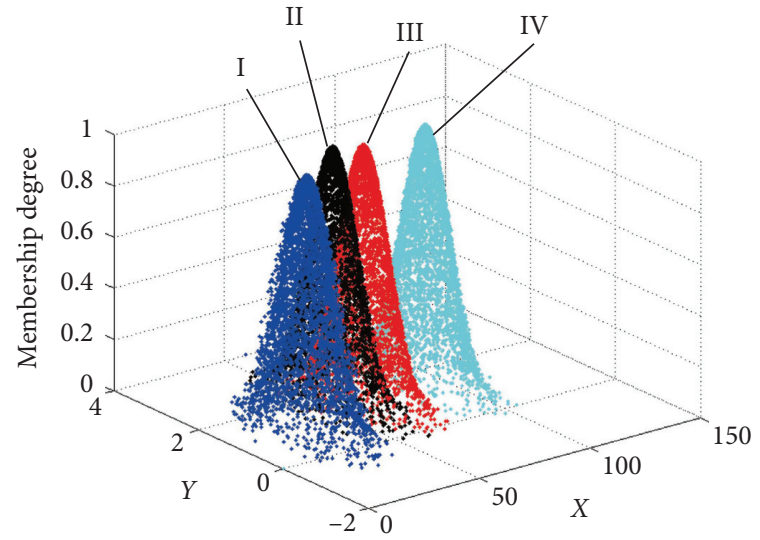

(a)

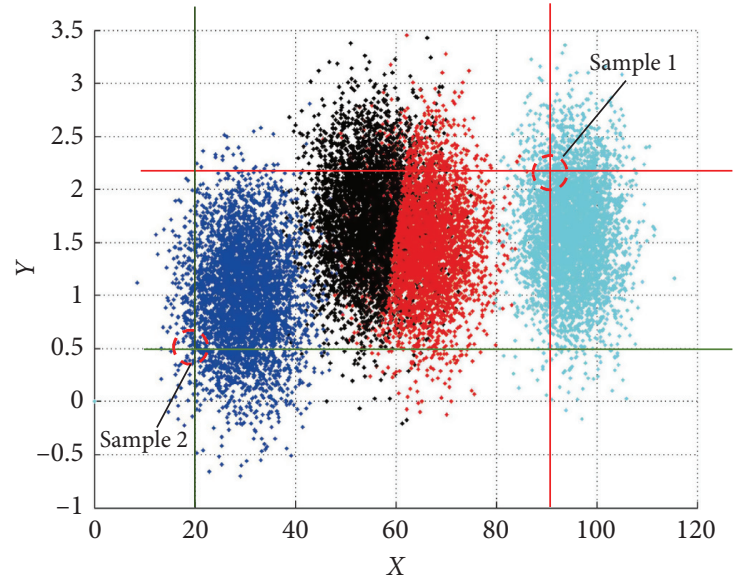

(b)

Figure 2: Two-dimensional cloud model with four levels of evaluation and its membership determination method. (a) 2D cloud models of different levels. (b) Two-index cloud model evaluation sample.

TABLE 1: The evaluation index of surrounding rock quality stability and the range of grading values.

\begin{tabular}{lccccc}
\hline Level & $\begin{array}{c}\text { Uniaxial compressive strength } \\
\text { UCS }(\mathrm{MPa})\end{array}$ & $\begin{array}{c}\text { Volume joint coefficient } \\
J v\left(\mathrm{~m}^{3}\right)\end{array}$ & $\begin{array}{c}\text { Integrity } \\
\text { coefficient } K v\end{array}$ & $\begin{array}{c}\text { Fracture water pressure } \\
P(\mathrm{MPa})\end{array}$ & $\begin{array}{c}\text { Rock mass quality index } \\
\text { BQ }(\mathrm{MPa})\end{array}$ \\
\hline I & $>60$ & $<3$ & $>0.75$ & $0.1-0.2$ & $>550$ \\
II & $60-30$ & $3-10$ & $0.75-0.55$ & $0.2-0.3$ & $550-451$ \\
III & $30-15$ & $10-20$ & $0.55-0.35$ & $0.4-0.5$ & $450-351$ \\
IV & $15-5$ & $20-3$ & $0.35-0.15$ & $0.7-0.9$ & $350-251$ \\
V & $\leq 5$ & $\geq 35$ & $\leq 0.15$ & 1.0 & $\leq 250$ \\
\hline
\end{tabular}

amount of information $C_{j}$, the calculation formula for determining the weight of the indicator is as follows:

$$
w_{j}=\frac{C_{j}}{\sum_{j=1}^{m} C_{j}}, \quad(j=1,2, \cdots, m) .
$$

2.2.3. Determination of Comprehensive Weights of Evaluation Indicators. Therefore, this paper combines the AHP and CRITIC methods to determine the weights of the multidimensional normal cloud model and can perform targeted evaluations on the surrounding rock during tunnel 
construction by mining the surrounding rock index data, thereby improving the stability evaluation method of the existing tunnel surrounding rock [15]. The combination of the analytic hierarchy process and the CRITIC method can highlight the advantages of subjective and objective weighting, make up for the shortcomings of a single weighting method, and effectively determine the importance of each evaluation of the surrounding rock stability indicator. The comprehensive weight determination formula used is as follows:

$$
w_{j}=\frac{w_{\mathrm{AHP}_{j}} w_{\mathrm{CRITIC}_{j}}}{\sum_{j=1}^{m} w_{\mathrm{AHP}_{j}} w_{\mathrm{CRITIC}_{j}}} .
$$

\section{Evaluation Framework of the Multidimensional Cloud Model for the Stability of the Surrounding Rock of the Complex Tunnel}

3.1. Calculation of the Digital Feature of the Surrounding Rock Quality Classification Index Cloud and Determination of Its Weight. The research on the evaluation index system of tunnel rock mass quality is relatively mature, and the selection of the index should consider the system, accessibility, difference, and representativeness, according to the work summary of surrounding rock stability evaluation research and field literature [8.9], and refer to the key indicators of surrounding rock stability classification in the Chinese standard "Engineering Rock Mass Classification Standard," and finally, selected rock mass uniaxial compressive strength (UCS) ( $\mathrm{MPa}$ ) and rock mass quality indicators BQ value, volume joint coefficient $J v$, integrity coefficient $K v$, and fracture water pressure are used as the index body of rock mass quality classification. In the rock mass quality evaluation, each factor index is divided into 5 grades from I to $\mathrm{V}$. The normalized values of the grading index dimensions are shown in Table 1.

This paper also uses calculation formulas (7)-(9) of the numerical characteristic value of the multidimensional cloud model in the literature [13] to calculate the stability classification index of the surrounding rock of the tunnel. The values are shown in the table.

$$
\begin{aligned}
& E x=\frac{C \max +C \min }{2}, \\
& E n=\frac{E x_{\max }}{3} .
\end{aligned}
$$

Generally speaking, the larger En is, the larger $\mathrm{He}$ is. $\mathrm{He}$ can be adjusted according to En:

$$
\mathrm{He} \in[0.01,0.5] \text {. }
$$

In the determination of the weight of the stability evaluation index of the surrounding rock of the tunnel, firstly, the analytic hierarchy process is used to determine the subjective weighting method, in which the discrimination matrix of the analytic hierarchy process based on expert scores is shown in the following equation:

$$
C_{A}=\left[\begin{array}{ccccc}
1 & 2 & 1 & 1 / 3 & 1 / 4 \\
1 / 2 & 1 & 1 / 2 & 1 / 4 & 1 / 6 \\
1 & 2 & 1 & 1 / 3 & 1 / 4 \\
3 & 4 & 3 & 1 & 1 / 3 \\
4 & 6 & 4 & 3 & 1
\end{array}\right] .
$$

After the consistency verification meets the requirements, the calculated subjective weight of the evaluation index system is

$$
w_{i}=\{0.1049,0.0605,0.1049,0.2517,0.4781\} .
$$

This paper collects typical samples of surrounding rock grading and stability evaluation during the engineering process as the objective samples of the CRITIC method to collect on-site sample indicators. Typical indicators are shown in Table 2.

According to the obtained sample index value, the objective weight vector of the index is calculated based on the CRITIC method:

$$
w_{j}=\{0.208,0.126,0.195,0.124,0.347\} .
$$

Formula (17) is used to calculate the comprehensive weight of the surrounding rock stability evaluation index, and finally, the comprehensive weight vector is calculated:

$$
w=\{0.088,0.031,0.083,0.126,0.672\} .
$$

Through the above calculations, Table 3 shows the comprehensive weights of the digital feature values and indicators of the multidimensional cloud model based on improved weighting.

3.2. Evaluation Framework of Surrounding Rock Stability of the Multidimensional Cloud Model. The calculation steps of the multidimensional normal cloud model for the stability of the surrounding rock are shown in Figure 3.

\section{Engineering Example Application}

This method is applied to the construction project of the Beijing-Shanghai Expressway and Jinan Expressway. The total length of the construction project is 21.95 kilometers, the bridge-tunnel ratio is $64.7 \%$, and there are 6 eight-lane tunnels on the whole line. The specific engineering conditions and surrounding rock characteristics are shown in Table 4 and Figure 4. The construction of the tunnel group is risky and difficult, and the scientific and accurate classification of the stability of the tunnel surrounding rock provides effective support to ensure the tunnel construction.

The evaluation of the multidimensional cloud model is carried out based on the surrounding rock sample indicators collected in the tunnel group. The actual values of the evaluation indicators of the tunnel surrounding rock are shown in Table 5.

According to the evaluation results of the multidimensional cloud model in the surrounding rock section in 
TABLE 2: Some site surrounding rock samples and index values.

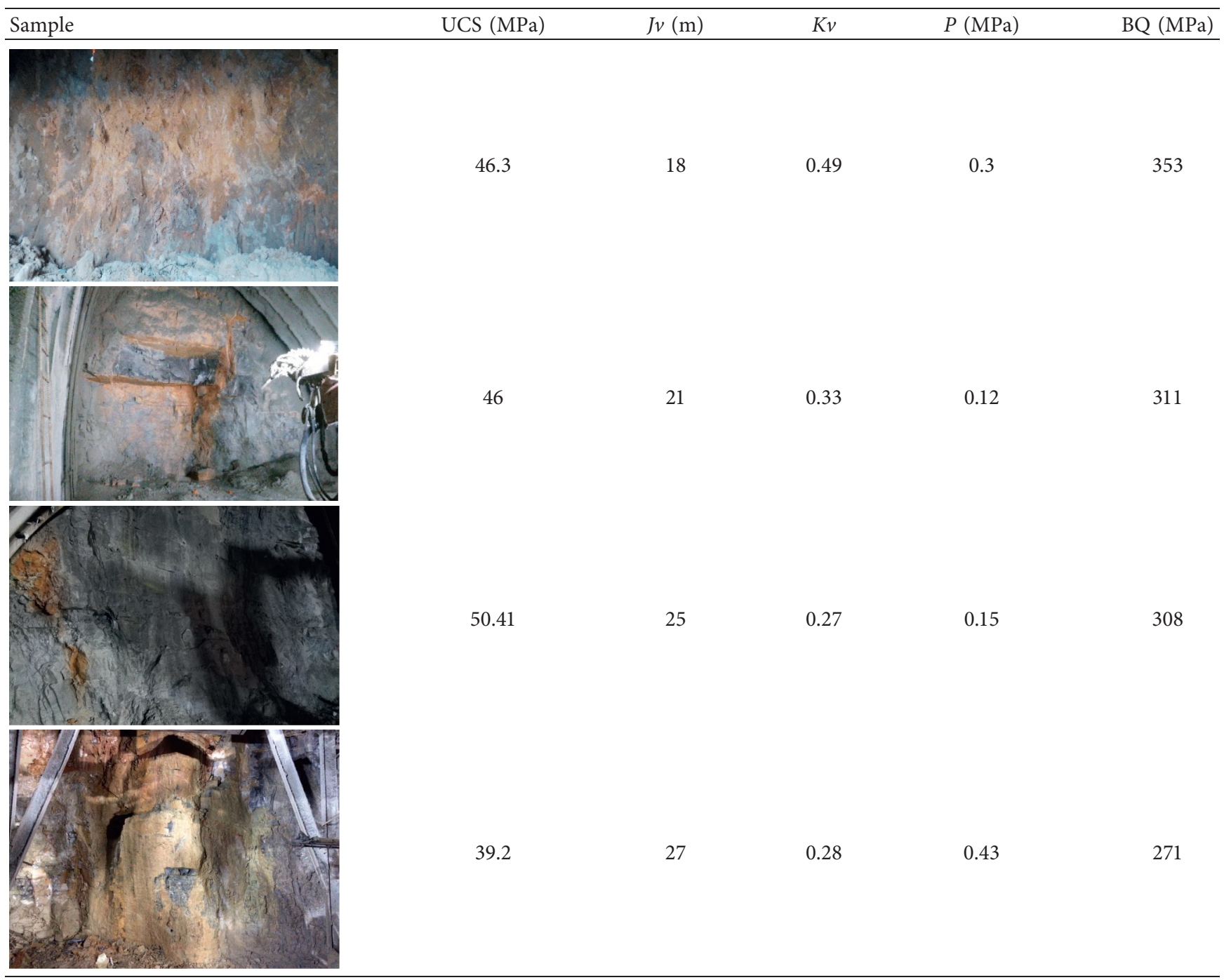

TABLE 3: The comprehensive weight distribution of digital feature values and indicators of the level II multidimensional cloud model of surrounding rock stability.

\begin{tabular}{lccccc}
\hline Digital features & UCS & $J v$ & $K v$ & & $P$ \\
\hline Ex & 45 & 6.5 & 0.65 & 0.25 & BQ \\
En & 15 & 2.1 & 0.21 & 0.08 & 1600 \\
He & 0.2 & 0.1 & 0.05 & 0.01 & 0.4 \\
Comprehensive weight & 0.088 & 0.031 & 0.083 & 0.126 & 0.672 \\
\hline
\end{tabular}

Table 6, it can be seen that the evaluation model in this article has some differences compared with the traditional fuzzy comprehensive evaluation method. From the analysis of the evaluation process of the first set of surrounding rock samples, the evaluation of the multidimensional cloud model is higher than the fuzzy comprehensive evaluation. This is because the multidimensional cloud model will perform positive cloud changes on the sample data to form a cloud drop cluster that conforms to the normal distribution.
Dituan, therefore, when calculating the membership level of the index, it will comprehensively consider the state of the index after the redistribution. In the fuzzy comprehensive evaluation method, an index value is used to determine a membership value, which is changed into a cloud with an index value, and then calculate the final index value through the membership degree distribution in the cloud group, thereby overcoming the influence of a single index on the comprehensive evaluation. 


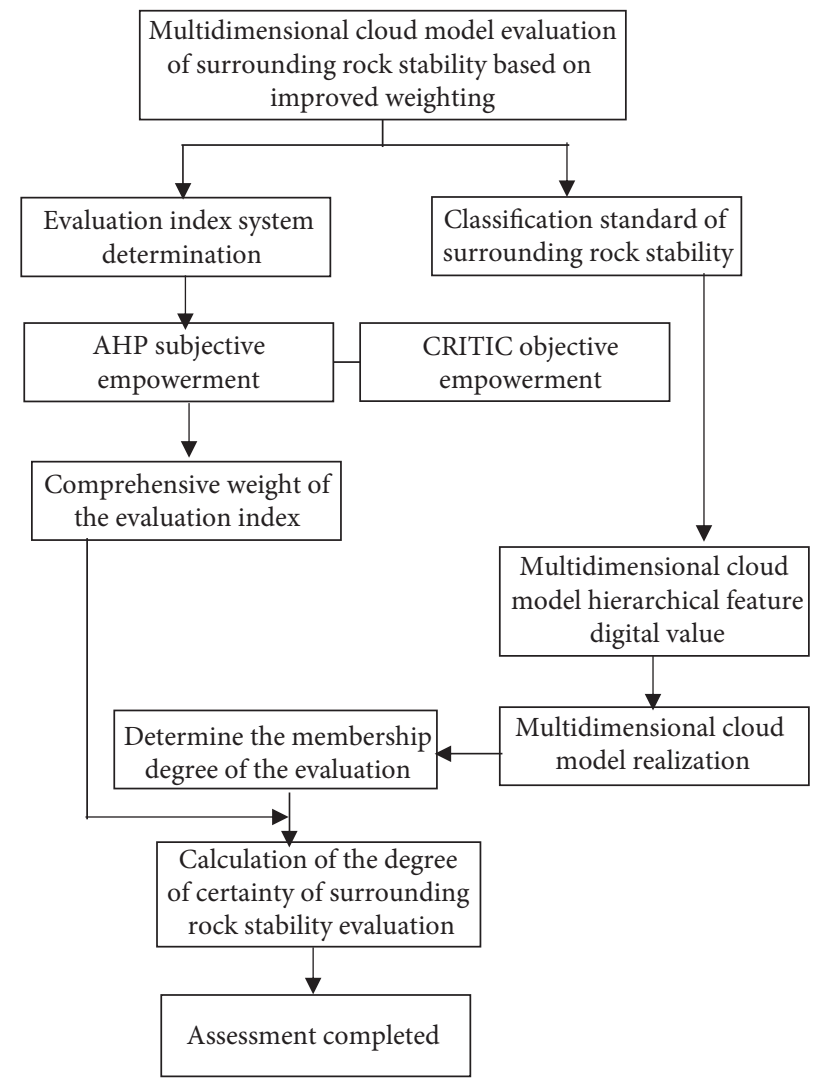

FIGURE 3: Multidimensional normal cloud model evaluation framework process of tunnel surrounding rock stability.

TABLE 4: Overview of the eight-lane tunnel group project.

\begin{tabular}{|c|c|c|}
\hline Tunnel name & Length $(\mathrm{m})$ & Project characteristics and main risks \\
\hline Laohushan Tunnel & $\begin{array}{l}\text { Left: } 1740 \mathrm{~m} \text {, right: } \\
1888 \mathrm{~m}\end{array}$ & $\begin{array}{l}\text { The tunnel is located on the side of the contact zone of the Upper and Lower Ordovician, there } \\
\text { are obvious intrusive rocks exposed, and the overall uniformity of the rock mass is poor. }\end{array}$ \\
\hline Daling Tunnel & $\begin{aligned} \text { Left: } 961.7 \mathrm{~m} \text {, right: } \\
965 \mathrm{~m}\end{aligned}$ & $\begin{array}{c}\text { Rocks are exposed in some sections of the tunnel site, joints and cracks are developed, and } \\
\text { rolling stones are seen at the foot of the slope. }\end{array}$ \\
\hline Xiaoling Tunnel & $\begin{array}{l}\text { Left: } 500 \mathrm{~m} \text {, right: } \\
500 \mathrm{~m}\end{array}$ & $\begin{array}{c}\text { There may be small dissolution fissures in local rock formations, with more developed } \\
\text { groundwater and joint fissures. }\end{array}$ \\
\hline Jian & Left: $3101 \mathrm{~m}$, right: & The tunnel body passes through multiple fault fracture zones and is affected by groundwater. \\
\hline Tunnel & $3085 \mathrm{~m}$ & There are many karst \\
\hline Longding Tunnel & $\begin{array}{l}\text { Left: } 2183 \mathrm{~m} \text {, right: } \\
2182 \mathrm{~m}\end{array}$ & $\begin{array}{l}\text { There are two squeezed and dense joints developed in the tunnel site area, and the joints } \\
\text { produced along the structure develop and form corrosion cracks, dissolved pores, and caves. }\end{array}$ \\
\hline Ganggou Tunnel & $\begin{array}{l}\text { Left: } 1105 \mathrm{~m} \text {, right: } \\
1091 \mathrm{~m}\end{array}$ & $\begin{array}{l}\text { The karst is developed, the fillings in the cave are prone to collapse when the cave is excavated, } \\
\text { and water in the cave will have a large short-term inrush in the rainy season. }\end{array}$ \\
\hline
\end{tabular}

The surrounding rock classification module integrates the surrounding rock classification evaluation results into the GIS map component to visually display the surrounding rock information. At the same time, the surrounding rock classification statistics along the tunnel are made to facilitate the understanding of the overall tunnel situation. Taking the Laohushan Tunnel as an example, the results of the surrounding rock classification applied to the GIS construction management platform are shown in Figure 5.

\section{Summary}

This article applies the objective weight and subjective weight determination method of the evaluation index system of the analytic hierarchy process and the CRITIC method to the comprehensive index weight in the process of surrounding rock stability evaluation. The determination of the comprehensive index weight can reflect the subjective experience of the evaluator. Judgment has 


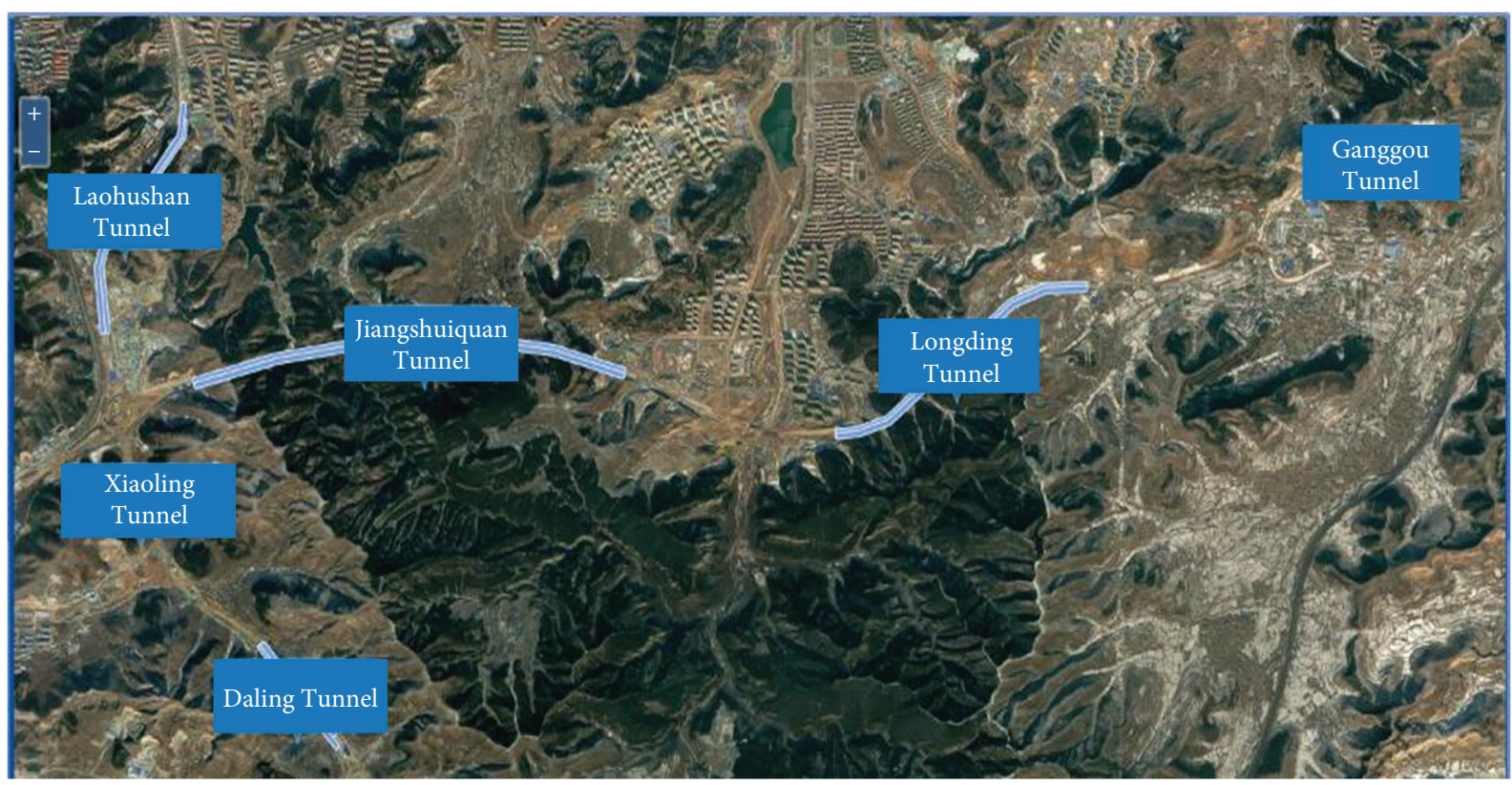

FIGURE 4: Overview of the topographic map of the eight-lane tunnel group.

TABLe 5: Measured values of the evaluation section indexes of the surrounding rock of the tunnel.

\begin{tabular}{lcccccc}
\hline Evaluation section & UCS $(\mathrm{MPa})$ & $J v(\mathrm{~m})$ & $K v$ & $P(\mathrm{MPa})$ & BQ $(\mathrm{MPa})$ & Description \\
\hline 1 & 43.02 & 27 & 0.25 & 0.11 & 285 & Broken surrounding rock \\
2 & 42.08 & 26 & 0.16 & 0.36 & 256 & Water seepage in the face \\
3 & 52.65 & 14 & 0.47 & 0.24 & 352 & The surrounding rock is relatively complete \\
4 & 50.91 & 32 & 0.19 & 0.13 & 290 & The surrounding rock is relatively complete \\
5 & 28.50 & 27 & 0.26 & 0.32 & 241 & Broken surrounding rock, seepage water \\
6 & 45.00 & 22 & 0.27 & 0.15 & 263 & Broken surrounding rock, seepage water \\
\hline
\end{tabular}

TABLE 6: Evaluation results of the multidimensional cloud model for the surrounding rock section.

\begin{tabular}{|c|c|c|c|c|c|c|c|}
\hline \multirow{2}{*}{ Evaluation paragraph } & \multicolumn{5}{|c|}{ Grade comprehensive certainty } & \multirow{2}{*}{ Method of this article } & \multirow{2}{*}{ Fuzzy comprehensive evaluation method } \\
\hline & I & II & III & IV & $\mathrm{V}$ & & \\
\hline 1 & 0.2825 & 0.5679 & 0.8648 & 0.5154 & 0.2334 & III & IV \\
\hline 2 & 0.4326 & 0.7765 & 0.3654 & 0.4014 & 0.2848 & II & II \\
\hline 3 & 0.1265 & 0.4813 & 0.447 & 0.6831 & 0.1962 & IV & $\mathrm{V}$ \\
\hline 4 & 0.6854 & 0.5486 & 0.7953 & 0.7663 & 0.8764 & $\mathrm{~V}$ & IV \\
\hline 5 & 0.5642 & 0.6046 & 0.7305 & 0.4536 & 0.1938 & III & III \\
\hline 6 & 0.2319 & 0.5427 & 0.6318 & 0.5831 & 0.7584 & V & V \\
\hline
\end{tabular}

strong objectivity, and empowerment is more reasonable and scientific. At the same time, the multidimensional cloud model evaluation method is used to replace the conventional cloud model evaluation method. The improved multidimensional cloud model after the matter element structure can not only integrate qualitative and quantitative indicators into a comprehensive evaluation model but also can reduce the influence of the state value of a single indicator on the overall evaluation model, the ambiguity and randomness of determining the stability level interval are reduced, and the reliability of the evaluation result is higher. Compared with the one-dimensional cloud model, the evaluation workload under the multidimensional model is smaller, and the evaluation process can be easily realized through programming. In addition, this article publishes the stability evaluation results of the surrounding rock of the tunnel through the GIS platform, which have practical significance for guiding the construction and development of the tunnel. 


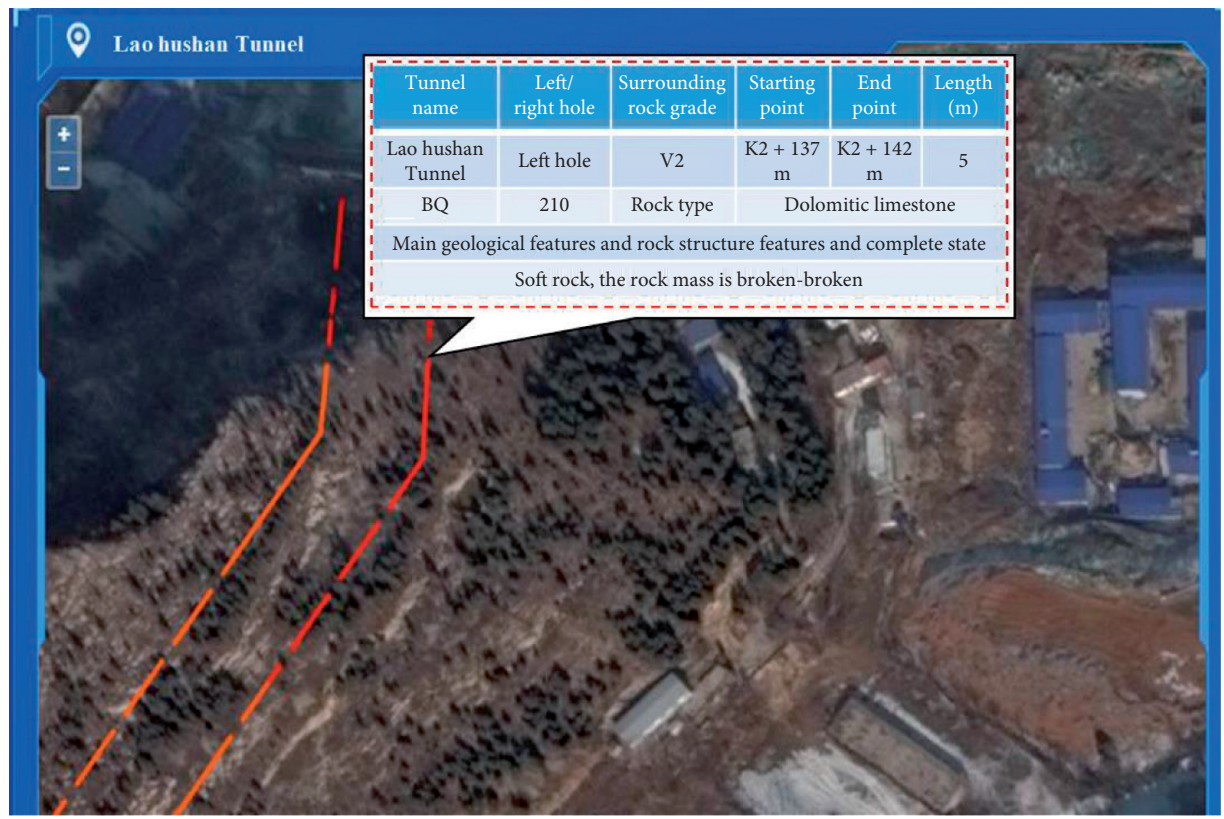

FIGURE 5: Application of surrounding rock classification results to the tunnel GIS construction management platform.

\section{Data Availability}

Some or all data, models, or codes that support the findings of this study are available from the corresponding author upon reasonable request.

\section{Conflicts of Interest}

The authors declare that they have no conflicts of interest.

\section{Acknowledgments}

This work was supported by the Key Laboratory of Geological Hazards on Three Gorges Reservoir Area (China Three Gorges University) (2018KDZ03) and the National Natural Science Foundation of China (no. 51879150).

\section{References}

[1] K. Ma, J. Zhang, Z. Zhou, and N. Xu, "Comprehensive analysis of the surrounding rock mass stability in the underground caverns of Jinping I hydropower station in Southwest China," Tunnelling and Underground Space Technology, vol. 104, Article ID 103525, 2020.

[2] Z. Tang, X. Liu, Q. Xu, C. Li, and P. Qin, "Stability evaluation of deep-buried TBM construction tunnel based on microseismic monitoring technology," Tunnelling and Underground Space Technology, vol. 81, pp. 512-524, 2018.

[3] H. Su, Z. Jiang, and H. Wu, "Study on a combined evaluation model of the stability of surrounding rock based on connection number," Highway Engineering, vol. 45, no. 1, pp. 18-22, 2020.

[4] S. Fang, H. Tian, D. Huang et al., "Study on classification of stability of surrounding rock using entropy weight method and multi-dimensional cloud model," Safety in Coal Mines, vol. 51, no. 1, pp. 229-232, 2020.
[5] X. Xue and M. Xiao, "Deformation evaluation on surrounding rocks of underground caverns based on PSO-LSSVM," Tunnelling and Underground Space Technology, vol. 69, pp. 171-181, 2017.

[6] S. Wu, S. Yang, and X. Du, "A model for evaluation of surrounding rock stability based on D-S evidence theory and error-eliminating theory," Bulletin of Engineering Geology and the Environment, vol. 80, no. 3, pp. 2237-2248, 2021.

[7] Q. Ren, L. Xu, A. Zhu et al., "Comprehensive safety evaluation method of surrounding rock during underground cavern construction," Underground Space, vol. 6, no. 1, pp. 46-61, 2021.

[8] L. Ran, Y. Yicheng, Z. Guangquan et al., "Grading prediction model of rockburst based on rough set-multidimensional normal cloud," Metal Mine, vol. 3, pp. 48-55, 2019.

[9] L. Zhong, Q. Xie, and Y. Luo, "Stability evaluation of tunnel surrounding rock based on game-extension theory," Journal of Safety Science and Technology, vol. 15, no. 1, pp. 56-61, 2019.

[10] J. Wang, Z. Zheng, Z. Li et al., "Evaluation of stability of tunnel surrounding rock based on extension cloud theory integrated with game theory," Railway Standard Design, vol. 62, no. 11, pp. 118-124, 2018.

[11] J. Zhou, "Stability analysis and evaluation index determination of surrounding rock of tunnel," Transportation Science \& Technology, vol. 6, pp. 110-113, 2018.

[12] R. Mou and Q. Cai, "Stability evaluation for the surrounding rock structure based on the normal cloud model and the rough set theory," Journal of Safety and Environment, vol. 18, no. 4, pp. 1251-1257, 2018.

[13] T. Zhou, J. Hu, and Y. Kuang, "Rock mass quality evaluation method and application based on fuzzy RES-multidimensional cloud model," The Chinese Journal of Nonferrous Metals, vol. 29, no. 8, pp. 1771-1780, 2019.

[14] J. O. B. U. Astronautics, "Threat assessment of air combat target based on RS-CRITIC," Journal of Beijing University of Aeronautics and Astronautics, vol. 46, no. 12, pp. 2357-2365, 2020.

[15] W. Qi, W. Li, Z. Li et al., "Rock mass quality evaluation of underground mine based on CRITIC-CW method," Gold Science and Technology, vol. 28, no. 2, pp. 264-270, 2020. 